University of Nebraska - Lincoln

DigitalCommons@University of Nebraska - Lincoln

8-1-2005

\title{
Selective Breeding for High Endurance Running Increases Hindlimb Symmetry
}

Theodore Garland Jr.

University of California, Riverside

Patricia W. Freeman

University of Nebraska-Lincoln, pfreeman1@unl.edu

Follow this and additional works at: https://digitalcommons.unl.edu/natrespapers

Part of the Natural Resources and Conservation Commons, and the Zoology Commons

Garland, Theodore Jr. and Freeman, Patricia W., "Selective Breeding for High Endurance Running Increases Hindlimb Symmetry" (2005). Papers in Natural Resources. 20.

https://digitalcommons.unl.edu/natrespapers/20

This Article is brought to you for free and open access by the Natural Resources, School of at DigitalCommons@University of Nebraska - Lincoln. It has been accepted for inclusion in Papers in Natural Resources by an authorized administrator of DigitalCommons@University of Nebraska - Lincoln. 


\title{
SELECTIVE BREEDING FOR HIGH ENDURANCE RUNNING INCREASES HINDLIMB SYMMETRY
}

\author{
Theodore Garland, JR. ${ }^{1}$ And Patricia W. Freeman ${ }^{2}$ \\ ${ }^{1}$ Department of Biology, University of California, Riverside, California 92521 \\ E-mail: tgarland@ucr.edu \\ ${ }^{2}$ School of Natural Resources and University of Nebraska State Museum, University of Nebraska, Lincoln, Nebraska 68588 \\ E-mail: pfreeman1@unl.edu
}

\begin{abstract}
Comparative studies provide correlational evidence of morphological adaptations for high locomotor performance, such as the classical indicators of cursoriality in mammals, long limbs and high metatarsal/femur ratios. More recently, enlarged femoral condyles have been suggested as an adaptation for high endurance running in the genus Homo. Asymmetry of locomotor appendages should adversely affect locomotor abilities, but this has not been studied in a rigorous evolutionary context. We used experimental evolution to test for morphological adaptations associated with high voluntary wheel running in selectively bred lines of mice. Surprisingly, the classical indicators of cursoriality had not evolved in concert with high activity levels. Instead, high runners had larger femoral condyles and reduced directional asymmetry of hindlimb bones. We hypothesize that greater limb symmetry and larger femoral heads are general adaptations associated with sustained, high-speed locomotion.
\end{abstract}

Key words.-Adaptation, aerobic capacity, artificial selection, asymmetry, cursoriality, directional selection, experimental evolution, locomotion.

Received April 1, 2005. Accepted June 6, 2005.

In this study we tested for cursorial adaptations in hindlimb bones of lines of house mice that had been selectively bred (11 generations) for high endurance running (see below and Swallow et al. 1998a; Garland 2003). By generation 10, daily wheel running (total revolutions) of the four replicate High Runner (HR) lines had reached $75 \%$ greater than the four random-bred Control lines, mainly by increased average speed, rather than time spent running (Swallow et al. 1998a). This elevated wheel running of the HR lines exceeded that exhibited by wild house mice bred and raised under the same conditions (Dohm et al. 1994; Garland 2003), and males from generation 10 (Swallow et al. 1998b) and 32 (Rezende et al. 2006) also exhibited a significantly elevated maximal aerobic capacity $\left(\mathrm{VO}_{2} \mathrm{max}\right)$ during forced treadmill exercise. Therefore, it seemed reasonable to expect that skeletal adaptations for high wheel running might also have evolved.

Comparative studies offer much correlational evidence of apparent morphological adaptations that enhance locomotor abilities, such as long legs and high metatarsal/femur (MTF) ratios in cursorial mammals (Garland and Janis 1993; Carrano 1999). Recent comparative studies have also identified apparent specialization for high endurance in the genus Homo, including enlarged femoral condyles (Bramble and Lieberman 2004 and references therein), which should reduce joint stress. Although widely used, comparative studies, by themselves, may not provide strong evidence concerning adaptation (Garland and Adolph 1994; Leroi et al. 1994; Garland et al. 2005). Experimental evolution approaches are one alternative, but have rarely been used in the context of functional morphology and biomechanics (Garland 2003; Swallow and Garland 2005). In this study, we hypothesized that the four replicate HR lines of mice would show longer hindlimbs, greater MTF ratios (Garland and Janis 1993; Carrano 1999), a larger femoral head (Bramble and Lieberman 2004), and reduced hindlimb DA and FA (Manning and Ockenden 1994), as compared with their four Control lines.

Aside from skeletal modifications that might facilitate various locomotor abilities, asymmetry of locomotor appendages should generally decrease biomechanical performance. Two types of asymmetry are most commonly studied (Palmer and Strobeck 2003). Directional asymmetry (DA) is defined simply as the right minus the left value of a trait. When average values for populations are computed, some degree of directional asymmetry is the rule for tetrapod limbs (including mice), and it is sometimes related to handedness (Lazenby 2002). Fluctuating asymmetry (FA) is defined as the absolute value of the right-left difference. Thus, FA indicates the magnitude of asymmetry, if any, but not its direction.

Fluctuating asymmetry has received much more attention than DA, and is usually interpreted as reflecting random developmental instability. Alternatively, FA may reflect habitually asymmetric behavior, such as gait asymmetries (Hallgrimsson 1998), or various other processes (Kellner and Alford 2003; Polack 2003). Regardless of its origins, experimental manipulations and studies of natural individual variation in birds indicate that wing FA can decrease locomotor performance (Swaddle 2003), and among individual lizards, femur FA is negatively associated with sprint speed (Martin and Lopez 2001). Moreover, FA is negatively associated with racing ability among individual thoroughbred horses (Manning and Ockenden 1994: DA was not reported). Therefore, we hypothesized that FA would be lower in the HR lines of mice as compared with their Control lines. Alternatively, no change might have occurred because the extent to which FA can evolve in response to selection is controversial (e.g., see Leamy et al. 2000).

Directional asymmetry can have important functional consequences and can be altered by selection (but see Leroi et al. 1994, p. 292 on dipterans), as in the grossly asymmetric external morphology of flatfishes (e.g., flounder) or the ear placement of many owl species (Norberg 1978). We are not aware of any studies of DA and locomotor abilities per se. Intuitively, however, and given the apparent negative effects of FA, DA might also be hypothesized to generally decrease locomotor abilities, although one can imagine that some types of running or jumping performance that involved frequent 
TABLE 1. Statistical comparisons of mice selectively bred for high endurance running (four replicate HR lines) with their four randomly bred control lines (with body mass used as a covariate and sex as an additional factor). All $P$-values represent one-tailed tests corresponding to a priori directional hypotheses. Body mass had a significant positive effect on both femoral condyle diameter and hindlimb length (both $P<0.0001$ ). Sex had a significant effect on hindlimb length (males shorter, two-tailed $P<0.0001$ ) and on metatarsal/femur ratio (males higher, two-tailed $P=0.0005$ ). The interaction between sex and linetype was not statistically significant for any trait (all $P \geq$ $0.3)$, but $P$-values shown are for models that included the interaction term.

\begin{tabular}{|c|c|c|c|c|c|c|}
\hline Trait $(n=138)$ & $\begin{array}{c}\text { Linetype } \\
F_{1,6} \\
\end{array}$ & Linetype $P$ & $\begin{array}{c}\text { Least squares } \\
\text { mean HR }\end{array}$ & $\begin{array}{l}\text { Least squares } \\
\text { mean control }\end{array}$ & $\begin{array}{c}\text { Least squares } \\
\text { SE HR }\end{array}$ & $\begin{array}{l}\text { Least squares } \\
\text { SE control }\end{array}$ \\
\hline Condyle diameter & 6.66 & 0.021 & 1.62 & 1.57 & 0.014 & 0.013 \\
\hline Hindlimb length & 0.00 & 0.477 & 42.3 & 42.3 & 0.269 & 0.267 \\
\hline Directional asymmetry in hindlimb length & 11.62 & 0.007 & 0.009 & $0.130 *$ & 0.0255 & 0.0241 \\
\hline Fluctuating asymmetry in hindlimb length ${ }^{1}$ & 3.46 & 0.056 & 0.356 & 0.418 & 0.0239 & 0.0228 \\
\hline
\end{tabular}

$* P=0.0016$ as compared with zero, indicating significant directional asymmetry is present in control lines (but not in HR lines, $P=0.7436$ ).

${ }^{1}$ Square-root transformed to improve normality; LS means and SEs are on square root scale.

turns could actually be enhanced by some degree of morphological asymmetry. As the HR lines are bred for essentially straight-line running performance, we hypothesized that their DA would also be reduced.

\section{Materials and Methods}

As described in detail elsewhere, beginning with outbred laboratory house mice of the Hsd:ICR strain, within-family selection for high voluntary wheel running was applied to four replicate lines (Swallow et al. 1998a; Garland 2003). In brief, mice were weaned at 21 days of age, then housed in same-sex groups of four/cage until 6-8 weeks of age, at which time they were housed individually and given access to computer-monitored wheels (1.12 m circumference) attached to standard housing cages. The selection criterion was total revolutions on days $5+6$ of a six-day exposure to wheels. Four additional lines, treated in identical fashion except that breeders were chosen randomly, served as Controls. Each line comprised 10 mating pairs per generation, sib-mating was disallowed, and ad lib food and water were always available.

Following routine wheel testing of all mice from generation 11, a random sample of males and females was chosen for the present study ( $n=138$ individuals in total). Because the sample was random, some of these individuals were necessarily required as breeders to produce the next generation. Mice were paired for breeding at approximately 10 weeks of age. After breeding, all individuals were housed individually without wheels until sacrifice by use of carbon dioxide at 232 days of age.

Mice were weighed, then skinned and eviscerated, and carcasses were air dried. Dried carcasses were placed in a colony of dermestid beetles, and bones were further cleaned manually as necessary. We followed a uniform protocol in skeletal preparation. Lengths of the femur, tibiafibula, and longest metatarsal were measured by one of us (PWF) to the nearest $0.01 \mathrm{~mm}$ with calipers, blind with respect to linetype. Each represents the average of three measurements done in quick succession, but the individual replicate measurements were not recorded. Hindlimb length was computed as the sum of femur + tibiafibula + longest metatarsal. The anterior-posterior diameter of the femoral condyle was also measured as above. For all traits, left and right sides were compared for asymmetry analyses and then averaged for size comparisons. Following standard conventions, directional asymmetry (DA) was computed as the right minus the left value of a trait; fluctuating asymmetry (FA) was computed as the absolute value of the right-left difference. Because FA can be affected by DA, we also computed the index FA2 of Palmer and Strobeck (2003), which is simply FA/DA.

As in previous comparisons of these replicate lines (e.g., see Swallow et al. 1998a, 2001, 2005; Koteja et al. 1999; Girard et al. 2001), Control and HR lines were compared by mixed model nested analysis of (co)variance with line as a random effect nested within linetype. Body mass was used as a covariate, and all analyses were performed with SAS Procedure Mixed (Release 8.02, available at: http:// v8doc.sas.com/sas/html.), which uses restricted maximum likelihood. The effect of linetype (Control lines vs. HR lines) was always tested over the variation among replicate lines with one and six degrees of freedom. Hence, any statistically significant linetype effect can truly be interpreted as a consistent (general) correlated response to the selective breeding for high wheel running (Henderson 1997). A probability of $<0.05$ was required to consider a difference statistically significant, and all tests of the linetype effect were one-tailed corresponding to directional hypotheses.

Sex was included as an additional fixed effect because of known differences in absolute levels of wheel running, running speed, body size, and many other traits (Swallow et al. 1998a, 2005; Garland 2003; Rezende et al. 2006). The effect of sex was tested over the sex $\times$ line(linetype) term (specified as a random effect) with 1 and $6 \mathrm{df}$. The interaction between sex and linetype was also tested over the sex $\times$ line(linetype) term with 1 and $6 \mathrm{df}$. In all analyses, residuals did not differ significantly from a normal distribution. Least-squares (adjusted) means and standard errors for HR and Control mice, correcting for variation in body mass, were also computed with SAS Procedure Mixed.

\section{RESULTS}

Mice from the HR lines had significantly larger femoral condyles (Table 1). Surprisingly, the classic indicators of cursoriality, hindlimb length, and MTF ratio, were not increased in HR mice.

With respect to hindlimb length symmetry, inspection of distributions of raw values and of residuals from the nested ANCOVA models indicated no substantial platykurtosis or bimodality, that is, no indication of antisymmetry (e.g., see 
Palmer and Strobeck 2003). Mice from the HR lines had significantly reduced DA of hindlimb length (Table 1). In fact, comparison of the adjusted mean DA for each linetype with the value of zero (which would indicate no DA) indicated that HR had no significant hindlimb DA, whereas Control, as expected from the literature, had significant DA (Table 1). Results for fluctuating asymmetry varied slightly depending on the index used and on what covariates were present in the model, but in all cases HR lines had lower values. For FA, the one-tailed $P$ values was 0.056 (Table 1 ). When mean hindlimb length was added as an additional covariate, it was not a significant predictor of FA (two-tailed $P=$ 0.228 ), but the $P$ value for linetype was reduced to 0.042 (one-tailed). For FA2, the linetype $P$ value was 0.059 , or, as with FA, 0.042 (one-tailed) when mean hindlimb length was included as a covariate (two-tailed $P$ for leg length $=0.143$ ).

\section{DisCUSSION}

Selective breeding is a powerful but underutilized approach for testing adaptive hypotheses about trait variation and covariation (Garland 2003; Swallow and Garland 2005). Of course, as with any model system, one must be cautious about extrapolating results to the real world. Several factors might account for discrepancies between our experimental results for house mice and expectations for cursorial animals based on comparative studies. First is the matter of definition. According to Hildebrand (1982): "Animals that travel far, fast, or easily on the ground are said to be cursorial"' (p. 437); "To contribute to speed, it is necessary to make the legs relatively long in relation to the other parts of the body"' ( $p$. $441)$; ". . . the distal segments of the legs usually lengthen more than the proximal segments" (p. 441); and "It is the ... metatarsals that lengthen most ...' (p. 442). Thus, multiple aspects of locomotor abilities are potentially involved with cursoriality. However, few of these have been studied quantitatively. Several studies have shown the leg length correlates with maximal sprint running speed among species of lizards (e.g., see Bonine and Garland 1999), and one study has shown this among species of large Carnivora and ungulates (Garland and Janis 1993). The latter study also found a significant relation between maximal speed and MTF ratio. However, our mice were selected for distance-running performance, not speed per se, and Harris and Steudel (1997) found no relation between residual leg length and either daily movement distance or home range area among species of Carnivora. Although the HR lines have increased distance running mainly by increasing speed, not the amount of time spent running, maximal wheel speeds are still much lower than maximal sprint speeds (Dohm et al. 1996; Swallow et al. 1998a; Koteja and Garland 2001; Rezende et al. 2006). On the other hand, female HR mice (males have not been studied) run more intermittently on wheels as compared with Controls, and this could be a mechanism for reducing the energetic cost of locomotion (Girard et al. 2001).

Second is the matter of size and scale. Some workers have suggested that cursorial adaptations are only likely in animals of relatively large body size (but see Steudel and Beattie 1993), and our mice are much smaller than the mammalian species usually examined with respect to cursoriality (Car- rano 1999; Biewener 2005). Third, the HR lines are selected for straight-line running on a wire mesh surface that does not contain irregularities or obstacles. This is very different from the natural world, where animals must move over or around micro- and macrotopographical features, and maneuverability may often be at a premium (LaBarbera 1983). Fourth, animals in nature must at least occasionally face food shortages, which could place a premium on energetic efficiency of locomotion, whereas our mice have ad lib food at all times, and energetics per se does not appear to be a major factor in the selection protocol (Koteja et al. 1999; Swallow et al. 2001).

Another possible reason that the classical cursorial adaptations have not evolved in the hindlimbs of HR mice is that they have instead evolved differences in running behavior (e.g., see Girard et al. 2001) and/or posture. Changes in posture might alter forces acting on the long bones (Biewener $2005)$ in a way that would ameliorate selection on their dimensions, but would not necessarily reduce stride frequency, which must be greatly increased in the HR lines, given that their increased running distance is mainly a function of increased speed, not amount of time spent running (Swallow et al. 1998a,b; Koteja et al. 1999; Girard et al. 2001; Koteja and Garland 2001; Swallow et al. 2001; Garland 2003; Rezende et al. 2006). We speculate that increased stride frequency might in turn lead to selection that favors larger femoral condyles. Presumably, larger condyles would reduce the area-specific effects of "wear and tear" that would be associated with higher stride frequencies and the associated increase in absolute numbers of footfalls (but see Farley and Taylor 1991).

Caveats notwithstanding, our results provide the first direct experimental evidence that reduced directional asymmetry of hindlimbs and larger femoral condyles may be primary morphological adaptations associated with the evolution of high sustained running ability. (On the other hand, under a sequential Bonferroni procedure [e.g., see Rice, 1989], the linetype difference in condyle diameter would not meet the criterion of $P<0.0125$ to be considered significant. We are curious to see whether significance increases in future generations.) Further, we suggest that the condylar signature may be a more reliable characteristic for investigating locomotion of fossil mammals than more traditional cursorial features (Carrano 1999). Our results also motivate the novel hypotheses that reduced limb DA may be a general adaptation associated with cursoriality, and that it will also be found when Homo, including human beings, are compared with other primates (cf. Bramble and Lieberman 2004).

\section{ACKNOWLEDGMENTS}

Funding for this project came from National Science Foundation grant IBN-0212567 to TG and a Layman Grant from the University of Nebraska-Lincoln to PWF. We thank R. Benedict for processing the mice. Our manuscript was improved by constructive comments from E. L. Brainerd, S. A. Kelly, C. A. Lemen, and two anonymous reviewers.

\section{Literature Cited}

Biewener, A. A. 2005. Biomechanical consequences of scaling. J. Exp. Biol. 208:1665-1676. 
Bonine, K. E., and T. Garland, Jr. 1999. Sprint performance of phrynosomatid lizards, measured on a high-speed treadmill, correlates with hindlimb length. J. Zool., Lond. 248:255-265.

Bramble, D. M., and D. E. Lieberman. 2004. Endurance running and the evolution of Homo. Nature 432:345-352.

Carrano, M. T. 1999. What, if anything, is a cursor? Categories versus continua for determining locomotor habit in mammals and dinosaurs. J. Zool., Lond. 247:29-42.

Dohm, M. R., C. S. Richardson, and T. Garland, Jr. 1994. Exercise physiology of wild and random-bred laboratory house mice and their reciprocal hybrids. Am. J. Physiol. 267(Regul. Integ. Comp. Physiol. 36):R1098-R1108.

Dohm, M. R., J. P. Hayes, and T. Garland, Jr. 1996. Quantitative genetics of sprint running speed and swimming endurance in laboratory house mice (Mus domesticus). Evolution 50: $1688-1701$.

Farley, C. T, and C. R. Taylor. 1991. A mechanical trigger for the trot-gallop transition in horses. Science 253:306-308.

Garland, T., Jr. 2003. Selection experiments: an under-utilized tool in biomechanics and organismal biology. Pp. 23-56 in V. L. Bels, J.-P. Gasc, A. Casinos, eds. Vertebrate biomechanics and evolution. BIOS Scientific Publishers, Oxford, U.K.

Garland, T., Jr., and S. C. Adolph. 1994. Why not to do two-species comparative studies: limitations on inferring adaptation. Physiol. Zool. 67:797-828.

Garland, T., Jr.,and C. M. Janis. 1993. Does metatarsal/femur ratio predict maximal running speed in cursorial mammals? J. Zool., Lond. 229:133-151.

Garland, T., Jr., A. F. Bennett, and E. L. Rezende. 2005. Phylogenetic approaches in comparative physiology. J. Exp. Biol. 208: In press.

Girard, I., M. W. McAleer, J. S. Rhodes, and T. Garland, Jr. 2001 Selection for high voluntary wheel running increases intermittency in house mice (Mus domesticus). J. Exp. Biol. 204: 4311-4320.

Hallgrimsson, B. 1998. Fluctuating asymmetry in the mammalian skeleton: evolutionary and developmental implications. Pp. 187251 in M. Hecht and M. Clegg, eds. Evolutionary biology. Plenum Press, New York.

Harris, M. A., and K. Steudel. 1997. Ecological correlates of hindlimb length in the Carnivora. J. Zool., Lond. 241:381-408.

Henderson, N. D. 1997. Spurious associations in unreplicated selected lines. Behav. Genet. 27:145-154.

Hildebrand, M. 1982. Analysis of vertebrate structure. 2d ed. John Wiley and Sons, New York.

Kellner, J. R, and R. A. Alford. 2003. The ontogeny of fluctuating asymmetry. Am. Nat. 161:931-947.

Koteja, P., and T. Garland, Jr. 2001. Forum: Response to R. Eikelboom. Anim. Behav. 61:F25-F26.

Koteja, P., J. G. Swallow, P. A. Carter, and T. Garland, Jr. 1999. Energy cost of wheel running in house mice: implications for coadaptation of locomotion and energy budgets. Physiol. Biochem. Zool. 72:238-249.
LaBarbera, M. 1983. Why the wheels won't go. Am. Nat. 121: 395-408.

Lazenby, R. 2002. Skeletal biology, functional asymmetry and the origins of "Handedness". J. Theor. Biol. 218:129-38.

Leamy, L. J., D. Pomp, E. J. Eisen, and J. M. Cheverud. 2000. Quantitative trait loci for directional but not fluctuating asymmetry of mandible characters in mice. Genet. Res. 76:27-40.

Leroi, A. M., M. R. Rose, and G. V. Lauder. 1994. What does the comparative method reveal about adaptation? Am. Nat. 143: 381-402.

Manning, J. T., and L. Ockenden. 1994. Fluctuating asymmetry in racehorses. Nature 370:185-186.

Martin, J., and P. Lopez. 2001. Hindlimb asymmetry reduces escape performance in the lizard Psammodromus aldirus. Physiol. Biochem. Zool. 74:619-624.

Norberg, R. A. 1978. Skull asymmetry, ear structure and function, and auditory localization in Tengmalm's owl, Aegolius funereus (Linne). Philos. Trans. Roy. Soc. Lond., B 282:325-410.

Palmer, A. R., and C. Strobeck. 2003. Nonlinear dynamics and developmental instability. Pp. 279-319 in M. Polak, ed. Fluctuating asymmetry analyses revisited. Oxford University Press, New York.

Polack, M., ed. 2003. Developmental instability: causes and consequences. Oxford Univ. Press, New York.

Rezende, E. L., S. A. Kelly, F. R. Gomes, M. A. Chappell, and T. Garland, Jr. 2006. Effects of size, sex, and voluntary running speeds on costs of locomotion in lines of laboratory mice selectively bred for high wheel-running activity. Physiol. Biochem. Zool. 79: In press.

Rice, W. R. 1989. Analyzing tables of statistical tests. Evolution 43:223-225.

Steudel, K., and J. Beattie. 1993. Scaling of cursoriality in mammals. J. Morphol. 217:55-63.

Swaddle, J. P. 2003. Fluctuating asymmetry, animal behavior, and evolution. Adv. Study Behav. 32:169-205.

Swallow, J. G., and T. Garland, Jr. 2005. Selection experiments as a tool in evolutionary and comparative physiology: insights into complex traits-An introduction to the symposium. Integr. Comp. Biol. 45:387-390.

Swallow, J. G., P. A. Carter, and T. Garland, Jr. 1998a. Artificial selection for increased wheel-running behavior in house mice. Behav. Genet. 28:227-237.

Swallow, J. G., T. Garland, Jr., P. A. Carter, W.-Z. Zhan, and G. C. Sieck. 1998b. Effects of voluntary activity and genetic selection on aerobic capacity in house mice (Mus domesticus). J. Appl. Physiol. 84:69-76.

Swallow, J. G., P. Koteja, P. A. Carter, T. Garland, Jr. 2001. Food consumption and body composition in mice selected for high wheel-running activity. J. Comp. Physiol. B 171:651-659.

Swallow, J. G., J. S. Rhodes, and T. Garland, Jr. 2005. Phenotypic and evolutionary plasticity of organ masses in response to voluntary exercise in house mice. Integr. Comp. Biol. 45:426-437.

Corresponding Editor: E. Brainerd 